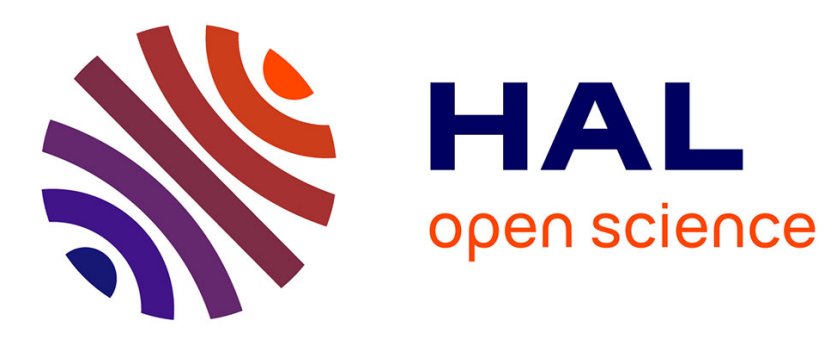

\title{
Artificial resonant crystals for hydroelastic waves
}

\author{
L. Domino, M. Fermigier, A. Eddi
}

\section{To cite this version:}

L. Domino, M. Fermigier, A. Eddi. Artificial resonant crystals for hydroelastic waves. Applied Physics Letters, 2020, 117 (6), pp.063701. 10.1063/5.0018823 . hal-02966867

\section{HAL Id: hal-02966867 \\ https: / hal.sorbonne-universite.fr/hal-02966867}

Submitted on 14 Oct 2020

HAL is a multi-disciplinary open access archive for the deposit and dissemination of scientific research documents, whether they are published or not. The documents may come from teaching and research institutions in France or abroad, or from public or private research centers.
L'archive ouverte pluridisciplinaire HAL, est destinée au dépôt et à la diffusion de documents scientifiques de niveau recherche, publiés ou non, émanant des établissements d'enseignement et de recherche français ou étrangers, des laboratoires publics ou privés. 


\title{
Artificial resonant crystals for hydroelastic waves
}

\author{
L. Domino, ${ }^{1}$ M. Fermigier, ${ }^{1}$ and $A$. Eddi $^{1,}$ a) \\ PMMH, CNRS, ESPCI Paris, Université PSL, Sorbonne Université, Université de Paris, F-75005, Paris, \\ France
}

(Dated: 29 July 2020)

Engineering the propagation of water waves is a crucial challenge for potential applications at oceanic scale. Despite their apparent complexity, they do not fundamentally differ from any other kind of physical wave. Similarly to recent approaches in optics or acoustics, we propose to artificially manufacture materials at the sub-wavelength scale to modify the propagation characteristics at will. We demonstrate that hydroelastic waves allow for straight-forward control of the medium properties as well as quantitative measurement of the full wave field. We investigate propagation in hydroelastic metamaterials made from periodic arrays of tunable resonators. We fully characterise the band structures of such materials, revealing the coexistence of Bragg and hybridisation band gaps. We also introduce a theoretical approach to model this complex system and predict its band structure. These unprecedented experimental and theoretical results reveal the possibility to efficiently control water waves at the laboratory scale.

Engineering water waves propagation is a crucial challenge as the potential applications at oceanic scale are tremendous. For example, they transport important quantities of energy, their instantaneous power being typically $40 \mathrm{~kW}$ per $\mathrm{m}$ of coast $^{1}$. As water waves are primarily waves, one can think of adapting strategies that have been implemented in optics, acoustics or electromagnetism. The recent development of metamaterials is thus particularly appealing ${ }^{2}$, as striking properties such as negative refractive index ${ }^{3}$, superlensing ${ }^{4,5}$ or cloaking $^{6}$ can be achieved. Some of these strategies have already been applied to water waves ${ }^{7-15}$, but they face practical constraints that limit both large scale applications and small scale laboratory studies. As a workaround, we propose to use a floating elastic membrane added at the air-water interface that provides a means to control the propagation medium and suppresses issues with free-surface (fig. 1 (a)). The resulting so-called hydroelastic waves ${ }^{16-19}$ are described by the following dispersion relation: in the limit of large wavenumber $k$, the angular frequency $\omega$ follows $\omega^{2}=D / \rho k^{5}$ with $D$ the flexural modulus, $\rho$ the fluid specific gravity (see fig. 1 (c) and suppl. mat. for a full derivation in the general regime). Such an approach allows for sub-wavelength focusing of the surface waves ${ }^{20}$. Here, we investigate experimentally concepts primarily inspired by solid state physics ${ }^{21}$ and extended later to optics and acoustics ${ }^{22}$ where a periodic modulation of the medium's properties prohibits the propagation in certain frequency ranges, called bandgaps. When the modulation is provided by scaterrers, one obtains Bragg gaps (fig. 1 (d)) whose frequency is selected by the pitch $a$ of the lattice. On the other hand, a random lattice of resonant scatterers exhibits a hybridisation band gap centered on the resonance frequency $f_{0}$, with a width set by the quality factor $Q$ of the resonators (fig. 1 (e)). Here, we obtain a medium's modulation by creating a regular lattice of resonant scaterrers in the membrane, namely circular holes, as shown in fig. 1 (b). We first study the behavior of a single resonator and then put hundreds of these on a regular square lattice. We measure very accurately the band structure of the lattice and we show the coexistence of differ-

\footnotetext{
a)Electronic mail: antonin.eddi@espci.fr
}

ent types of bandgaps, namely Bragg bandgaps and hybridisation bandgaps, due to scattering in the structure and local resonances, respectively (fig. 1 (f)). We then predict these dispersion relations with a theoretical approach combining Fano resonance and multiple scattering.
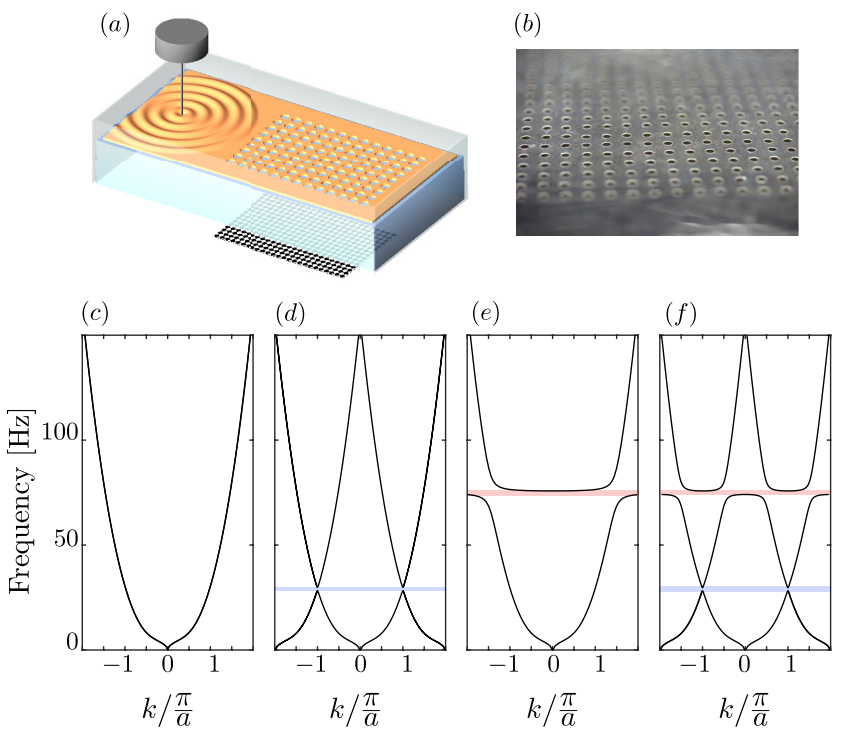

FIG. 1. (a) Sketch of the experimental set-up, with a perforated floating membrane and a vibration exciter (see methods for details). (b) Detail of one of the resonant crystal studied. The diameter of each perforation is $4 \mathrm{~mm}$ and the distance between them is $1 \mathrm{~cm}$. (c) Dispersion relation of hydro-elastic waves propagating in a floating membrane $\left(\rho=970 \mathrm{~kg} / \mathrm{m}^{3}, E=1.47 \pm 0.09 \mathrm{MPa}, h=300 \mu \mathrm{m}\right)$. (d) Dispersion relation for hydroelastic waves with a square lattice of scatterers with lattice constant $a$. The lattice periodicity implies the existence of a Bragg band gap, here denoted in blue, and whose size depends on the cross section of each scatterer. (e) Dispersion relation for hydroelastic waves with resonators randomly distributed in space. Here the size of the gap, denoted in red, depends on the quality factor $Q$ of the resonator, while its central frequency is set by the resonance frequency $f_{0}$. (f) Dispersion relation for hydroelastic waves with resonators distributed on a regular lattice. It exhibits both Bragg and hybridisation band gaps, with frequencies respectively selected by lattice pitch $a$ and the resonance frequency $f_{0}$. 
The experimental setup we use is similar to what we described previously ${ }^{20}$. We fill a glass tank $(80 \mathrm{~cm} \times 40 \mathrm{~cm} \times$ $20 \mathrm{~cm}$ ) with water to a depth of $h_{0}=16.5 \mathrm{~cm}$. We cover the water surface with an elastic sheet of thickness $e=300 \mu \mathrm{m}$ and dimensions $75 \mathrm{~cm} \times 35 \mathrm{~cm}$. It is made of an optically transparent silicone rubber, which has a Young's modulus of $E=1.47 \pm 0.09 \mathrm{MPa}$, a density of $\rho_{s}=970 \mathrm{~kg} / \mathrm{m}^{3}$ and a Poisson's ratio of $v=0.5$. This elastic film floats freely at the surface of water so that the mechanical tension $T$ applied to it reduces to the water surface tension $T=\sigma \simeq 50 \mathrm{mN} / \mathrm{m}$. We then structure the elastic sheet by punching circular holes (from one to up to several hundreds). We typically use perforations of diameter $3 \mathrm{~mm}$ or $4 \mathrm{~mm}$, and the distance between each perforation is about $1 \mathrm{~cm}$. The waves are generated using a vibration exciter (Bruel and Kjaerr 4809), driven by a waveform generator and a power amplifier. We work with small amplitudes $(\zeta \ll \lambda$ and $\zeta \ll e$ ) to ensure the waves are in the linear regime and that the thin membrane limit is valid ${ }^{23}$. To characterize the full frequency response of the artificial crystal we generated frequency sweeps between $2 \mathrm{~Hz}$ and $150 \mathrm{~Hz}$ at a rate of about $0.3 \mathrm{~Hz} / \mathrm{sec}$, which we checked to be small enough not to bias the measurement.

The wave fields were measured using standard synthetic Schlieren optical techniques ${ }^{24,25}$. This consists in measuring the apparent deformation of a known pattern due to refraction at the liquid-air surface. We take images of the pattern from the top, through the tank, the water and the polymer. Using ray optics, one can calculate the local slope at the interface $\theta$ as a function of the apparent displacement $\delta \mathbf{r}$ :

$$
\theta=-\frac{\delta \mathbf{r}}{h^{*}} \quad, \text { where } \frac{1}{h^{*}}=\frac{1}{\left(1-\frac{n_{\text {air }}}{n_{\text {water }}}\right) \cdot h_{0}}-\frac{1}{H}
$$

and $h_{0}$ is the water depth, $H$ the distance between the pattern and the camera, $n_{\text {air }}$ is the air refractive index and $n_{\text {water }}$ that of water. After reconstruction, we obtain 2D elevation fields ( 255 X 255 points) and we are able to measure amplitudes down to $\zeta=1 \mu \mathrm{m}$ (see Supp. Video 5 (multimedia view)). We then use Fourier filtering both in time and space to extract the spectra in the direction of propagation at a given frequency. We then concatenate this information for each frequency so as to extract the dispersion maps.

To understand how waves propagate in the artificial medium we have created, we first investigate the frequency response of a single perforation. We punch one circular hole with diameter $1 \mathrm{~cm}$ in the middle of a homogeneous elastic sheet and send plane waves towards it, as schematically shown in figure 2(a). We send plane waves between $5 \mathrm{~Hz}$ and $60 \mathrm{~Hz}$ on the perforation, and measure the surface elevation inside the perforation as well as around it, on a distance of about 5 $\mathrm{cm}$. An example of measured height field is shown in figure 2(b), where we denote the edge of the cavity with a red circle. We see that the amplitude inside the perforation is larger than in the sheet, which is expected for a free surface. We then average the amplitude over the whole cavity surface to analyze the frequency response and identify possible resonances. We report in figure 2(c) (top panel) the height fields measured experimentally for five frequencies $(16.6 \mathrm{~Hz}, 25.9 \mathrm{~Hz}, 33.4$,
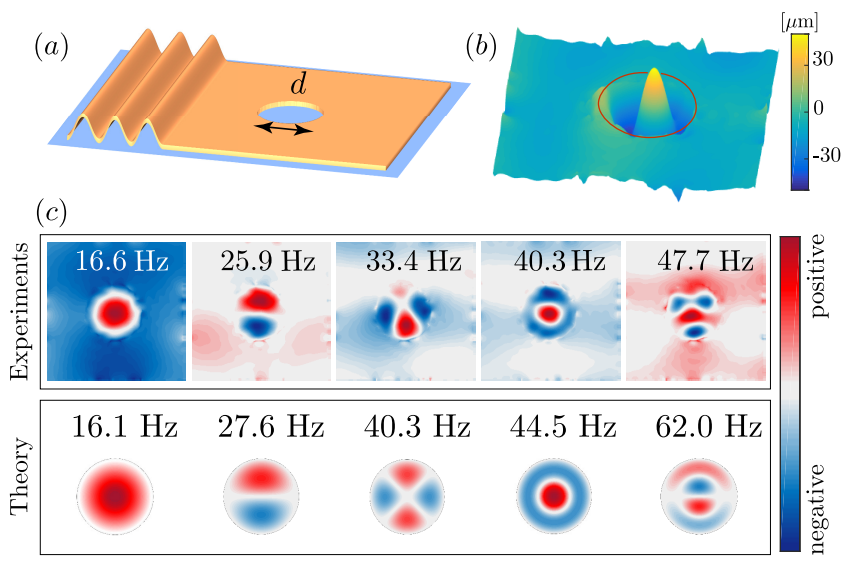

FIG. 2. (a) Sketch of the floating membrane with a single perforation and an incident plane wave. (b) Height field measured experimentally at $45 \mathrm{~Hz}$, showing the edge of the perforation of diameter 1 $\mathrm{cm}$ (red circle). (c) Comparison between experimental (upper panel) and analytical (lower panel) modes in the circular cavity. Results are normalised by their individual maximum amplitude.

$\mathrm{Hz}, 40.3 \mathrm{~Hz}$ and $47.7 \mathrm{~Hz}$ ) corresponding to peaks in the averaged frequency response of the hole. We compare them to the expected modes for a circular cavity with rigid walls and Dirichlet boundary conditions. The eigenmodes for the wave equation in a circular cavity are Bessel functions of the first kind that oscillate at the frequency $\omega=\frac{v_{\varphi} \alpha_{m n}}{R}$, where $\alpha_{m n}$ is the $\mathrm{n}^{\text {th }}$ zero of the Bessel function $J_{m}, v_{\varphi}$ is the phase velocity of the waves and $R$ is the radius of the hole (see Suppl. Mat.). The dispersion relation valid inside the perforation is that of gravity capillary waves ${ }^{26}$. We represent these Bessel functions in the lower panel of figure 2(c), where we only show the modes [0,1], [1,1], [2,1], [0,2] and [1,2] (from left to right). We observe an excellent agreement with the measured height field, suggesting that the perforations behave as circular resonant cavities indeed. However, we denote a discrepancy between some of the predicted and the measured frequencies. This might be due to the fact that the assumption of rigid walls and of fixed boundary conditions is clearly not met here: the outside membrane is deformed by the incoming wave, and the contact line pins at different heights at the edge of the hole. Despite this disparity, the perforations in the membrane behave, as we expected, as resonators with eigenfrequencies tuned by their diameter.

We now study wave propagation in a material made of $10 \times 20$ perforations organised on a square lattice with spacing $a$ as shown schematically in figure 3(a). We excite this artificial crystal with quasi plane waves between $5 \mathrm{~Hz}$ and $140 \mathrm{~Hz}$, and record the height field for each frequency. We show in figure 3(b) a typical wave field measured at $60 \mathrm{~Hz}$ for a lattice made of holes of diameter $4 \mathrm{~mm}$ and with a lattice constant $a=1 \mathrm{~cm}$. At this frequency, we observe the coexistence of two wavelengths in the crystal : one short wavelength (about $1.2 \mathrm{~cm}$ ), equal to the one in the homogeneous elastic sheet and which propagates from left to right, and one larger wavelength (about $4.2 \mathrm{~cm}$ ), that propagates from right to left (see Supp. Video 5 (Multimedia view)). To capture the behav- 

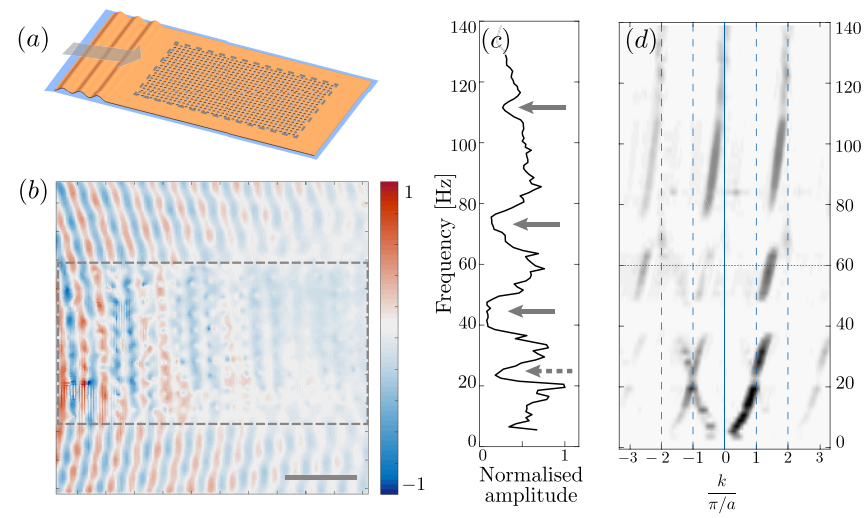

FIG. 3. (a) Sketch of the experiment showing $10 \times 20$ circular perforations organised on a square lattice. The grey dashed line denotes the edge of the crystal. (b) Wave field obtained at $60 \mathrm{~Hz}$. The square crystal is delimited by the dashed line. Waves propagated from left to right (see Supp. Video 6 (Multimedia view)). The scale bar is $5 \mathrm{~cm}$. (c) Amplitude measured inside the crystal (averaged over a large area avoiding the edges) as a function of frequency. (d) Map of the dispersion relation measured in the crystal (see text for details). The vertical dashed lines represent the edges of the Brillouin zones and the horizontal line corresponds to the frequency of the wavefield represented in (b).

ior of the perforated lattice on the entire frequency range we tested, we measured the amplitude of the waves averaged over a large area in the lattice (avoiding the edges). In other words, we are computing the effective density of states of our system. We plot in figure 3(c) this average amplitude against the frequency. We see several local minima in the curve, meaning that for some frequencies (denoted by arrows in figure 3(c)), the wave amplitude in the lattice decreases significantly.

To better understand the origin of these gaps and of the counter propagating wave, we perform 2D Fourier transforms at each frequency to isolate the spatial spectra of the waves propagating in the lattice. We only keep wavenumbers in the direction of propagation of the incoming wave $\mathrm{e}^{27}$, we can then build a map of the experimental dispersion relation by 'stacking' these spatial spectra for all frequencies. This dispersion relation is presented in figure 3(d), where we draw vertical dashed lines at the edge of each Brillouin zone. The pixel color denotes the intensity for the signal, so that we can recover the band structure of the lattice from the lines seen in the figure. We can only measure curves with positive slopes as they are the ones corresponding to a positive group velocity. We attribute the apparent negative slope at low frequency and negative wavenumber to reflexion on the right wall of the water tank. The band structure is not continuous : bands with very low amplitude can be seen around $22 \mathrm{~Hz}, 42 \mathrm{~Hz}, 70 \mathrm{~Hz}$ and $110 \mathrm{~Hz}$. When comparing the band structure (fig. 3(d)) to the frequency spectrum (fig. 3(c)), one can see the bandwidth of these gaps. The first gap, around $22 \mathrm{~Hz}$, has a relatively small bandwidth, and corresponds to the avoided crossing of two bands at the edge of the first Brillouin zone ; it is a Bragg band gap. The other three gaps at higher frequencies $(42 \mathrm{~Hz}, 70 \mathrm{~Hz}$ and $110 \mathrm{~Hz}$ ) are markedly different : their band- width is larger, and the orientation of the bands is opposite to that of the Bragg bandgaps (the curvature of two branches on each side of the gap now have the same sign). These are the hybridisation bandgaps due to the resonances of each cavity. Last, we can go back to our experimental wavefield (fig. 3(b)) taken at $60 \mathrm{~Hz}$ and explain the two wavelengths we observe. The forward propagating wave corresponds to the band on the right of the axis $k=0$, which has the same wavelength as the waves in the homogeneous sheet (outside the dashes rectangle in fig. 3(b)). The backward propagating wave corresponds to the branch on the left, for which $k$ is negative. Because $v_{\varphi}=\frac{\omega}{k}$ is negative (while $v_{g}=\frac{d \omega}{d k}$ stays positive) the wave propagates backwards, here from right to left.

By changing the properties of the lattice (namely the lattice constant and the size of the perforations) we can now design lattices with tunable bandgaps. We report in figure 4 (left panels) the dispersion relations measured for three different lattices. Here we symmetrise the map with respect to the axis $k=0$ to highlight the band structure (the band structure in figure 4(a) is the symmetrised version of the one in figure 3(d)). We first study the effect of changing the size of the perforations : we create a lattice with smaller circular holes (diameter $3 \mathrm{~mm}$ ) with the same spacing $a=1 \mathrm{~cm}$. Its measured dispersion relation is shown in figure 4(b). As expected the Bragg bandgap is located at the same frequency, ie. $22 \mathrm{~Hz}$, since it is set by the lattice constant only. However, the hybridisation bandgaps open at higher frequencies, here $68 \mathrm{~Hz}$ and $100 \mathrm{~Hz}$.

Alternatively, we can change the distance between the perforations without varying the size of the holes. To test this we created a lattice made of perforations of diameter $4 \mathrm{~mm}$ distant of $8.25 \mathrm{~mm}$ (fig. 4(c)). It exhibits a Bragg bandgap at the edge of the first Brillouin zone at about $38 \mathrm{~Hz}$, higher than the previous value of $22 \mathrm{~Hz}$. However, one would expect the hydridization bandgaps to appear at the same frequency whatever the spacing between the perforations, whereas here we observe the opening of the two hybridisation bandgaps at higher frequencies $(60 \mathrm{~Hz}$ and $82 \mathrm{~Hz})$ than the frequencies measured for a larger lattice constant $(42 \mathrm{~Hz}$ and $72 \mathrm{~Hz}$, see fig. 4(a)). Here again, we attribute this difference to near field coupling between resonators, which shifts their resonance frequencies, a phenomenon already observed in optics ${ }^{28}$ or with EM waves ${ }^{29}$. We are thus able to create materials with several bandgaps, whose frequency we can tuned at will.

We work with 2D materials but we only probe them in one direction. We can thus use the framework of 1D materials to model wave propagation in our system. Inspired by previous work on acoustics metamaterials ${ }^{22,30,31}$, we use resonant point scatterers to model the circular perforations in the floating membrane ${ }^{32}$, assuming that their typical size is sufficiently small compared to the wavelength. For each resonance, their frequency response is described by a Lorentz function, and each object has several eigenmodes, which leads to several resonance frequencies. With this we can write the amplitude and phase of the wave $T$ transmitted downstream of the resonator. We combine this approach to multiple scattering theory by using the transfer matrix formalism (see model in Suppl. Mat. for details). Seeking solutions in the form of Bloch waves, and including dispersion by introducing the 

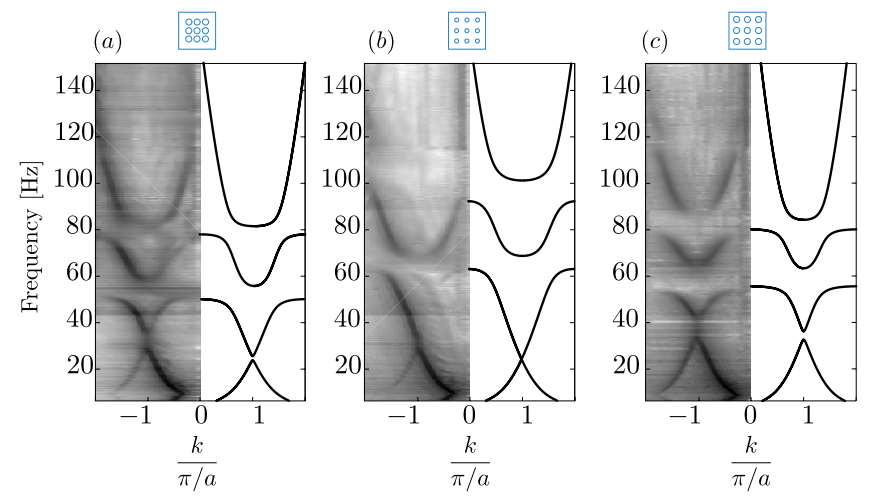

FIG. 4. Band structures measured for three different lattices with different lattice constants $a$ and different hole diameters $d$, as schematically shown in the sketches above each band structure. The left panels correspond to the experimental measurements, the right panels to the prediction from eq. 2 with the following parameters: (a) $\mathrm{a}=1 \mathrm{~cm}$, $\mathrm{d}=4 \mathrm{~mm}, \omega_{0}=50 \mathrm{~Hz}, Q_{0}=10, \omega_{1}=80 \mathrm{~Hz}$ and $Q_{1}=20$, (b) a $=$ $1 \mathrm{~cm}, \mathrm{~d}=3 \mathrm{~mm}, \omega_{0}=65 \mathrm{~Hz}, Q_{0}=10, \omega_{1}=100 \mathrm{~Hz}$ and $Q_{1}=10$ (c) a $=8.25 \mathrm{~mm}, \mathrm{~d}=4 \mathrm{~mm}, \omega_{0}=55 \mathrm{~Hz}, Q_{0}=10, \omega_{1}=80 \mathrm{~Hz}$ and $Q_{1}=20$.

hydroelastic wave velocity $c(\omega)$ obtained from the dispersion relation ${ }^{20}$, we can compute the analytical band structure of our systems :

$$
\cos k a=\operatorname{Re}\left(\frac{1}{T} e^{-j \frac{\omega}{c} a}\right) .
$$

We solve this equation numerically and we show in figure 4 (right panels) the resulting band structures, shown in direct comparison with the measured band structure. One can readily see that our simple model captures well the characteristics of the dispersion relation : the bandgaps are correctly predicted, as well as the band curvature on each side of the gap, confirming the nature of the latter. We can even understand the propagation of waves in $2 \mathrm{D}$, which we study by exciting a crystal with a plane wave with a non-zero incidence. We illustrate this further in the Suppl. Mat., where we separate different modes to highlight the effect of the periodicity of the crystal.

In this work, we introduce a macroscopic experimental approach that allows for direct tuning and measurement of wave propagation inside artificial resonant crystals. Using hydroelastic waves, we are able to design periodic structures exhibiting both Bragg and hybridisation band gaps. We fully characterize the properties of these artificial structures and propose a modelling that describes our experimental findings. Notably, our system offers promising perspectives in terms of applications : scaling up this approach of hydroelastic waves at oceanic scale could offer unprecedented control of water waves in order to protect coast or concentrate water wave energy upon harvesting devices.

In addition, our experimental system opens enticing perspectives to study wave propagation, to reveal analogies with solid state physics and more generally to uncover new phenomena. Hydroelastic waves could be used to probe systematically the physics of $2 \mathrm{D}$ periodic media as well as to design macroscopic topological materials. In particular, the usual bottlenecks met when attempting to control electromagnetic waves (i.e. controlling the medium properties, manufacturing objects at the scale of the wavelength, measuring quantitatively the wave amplitude) are readily circumvented by the use of macroscopic water waves.

Supplementary material. - See supplementary material for the complete description of the experimental set-up, the detailed presentation of the theoretical approach we developed and for an example of a refraction at a square lattice interface with non-zero incidence. Supplementary videos show the measuring technique used (Supp. Video 5 (multimedia view)) and the propagation of waves in a square lattice for a zeroangle incidence (Supp. Video 6 (multimedia view)) and for a non-zero incidence (Supp. Video 7 (multimedia view)) .

Acknowledgments. - The authors would like to thank E. Fort, S. Yves, N. Kaina, F. Lemoult and Y. Couder for fruitful discussions.

The data that support the findings of this study are available from the corresponding author upon reasonable request.

${ }^{1}$ E. Callaway, "To catch a wave: ocean wave energy is trying to break into the renewable-energy market, but many challenges remain," Nature 450, 156-160 (2007).

${ }^{2}$ P. A. Deymier, Acoustic metamaterials and phononic crystals, Vol. 173 (Springer Science \& Business Media, 2013).

${ }^{3}$ D. R. Smith, J. B. Pendry, and M. C. Wiltshire, "Metamaterials and negative refractive index," Science 305, 788-792 (2004).

${ }^{4}$ F. Lemoult, G. Lerosey, J. de Rosny, and M. Fink, "Resonant metalenses for breaking the diffraction barrier," Phys. Rev. Lett. 104, 203901 (2010).

${ }^{5} \mathrm{X}$. Zhang and Z. Liu, "Superlenses to overcome the diffraction limit," Nature materials 7, 435 (2008).

${ }^{6}$ J. B. Pendry, D. Schurig, and D. R. Smith, "Controlling electromagnetic fields," science 312, 1780-1782 (2006).

${ }^{7}$ M. Belzons, P. Devillard, F. Dunlop, E. Guazzelli, O. Parodi, and B. Souillard, "Localization of surface waves on a rough bottom: Theories and experiments," EPL (Europhysics Letters) 4, 909 (1987).

${ }^{8}$ M. Belzons, V. Rey, and E. Guazzelli, "Subharmonic bragg resonance for surface water waves,” EPL (Europhysics Letters) 16, 189 (1991).

${ }^{9}$ M. Berry, R. Chambers, M. Large, C. Upstill, and J. Walmsley, "Wavefront dislocations in the aharonov-bohm effect and its water wave analogue," European Journal of Physics 1, 154 (1980).

${ }^{10}$ M. Farhat, S. Enoch, S. Guenneau, and A. Movchan, "Broadband cylindrical acoustic cloak for linear surface waves in a fluid," Physical review letters 101, 134501 (2008).

${ }^{11}$ C. Berraquero, A. Maurel, P. Petitjeans, and V. Pagneux, "Experimental realization of a water-wave metamaterial shifter," Physical review E 88, 051002 (2013).

${ }^{12}$ Z. Wang, P. Zhang, X. Nie, and Y. Zhang, "Focusing of liquid surface waves by gradient index lens," EPL (Europhysics Letters) 108, 24003 (2014).

${ }^{13}$ X. Hu, Y. Shen, X. Liu, R. Fu, J. Zi, X. Jiang, and S. Feng, "Band structures and band gaps of liquid surface waves propagating through an infinite array of cylinders," Physical Review E 68, 037301 (2003).

${ }^{14}$ M. Torres, J. Adrados, F. M. de Espinosa, D. Garcia-Pablos, and J. Fayos, "Parametric bragg resonances in waves on a shallow fluid over a periodically drilled bottom," Physical Review E 63, 011204 (2000).

${ }^{15}$ R. B. Elandt, M. Shakeri, and M.-R. Alam, "Surface gravity-wave lensing," Physical Review E 89, 023012 (2014).

${ }^{16}$ V. A. Squire, J. P. Dugan, P. Wadhams, P. J. Rottier, and A. K. Liu, "Of ocean waves and sea ice," Annual Review of Fluid Mechanics 27, 115-168 (1995).

${ }^{17}$ V. Squire, "Of ocean waves and sea-ice revisited," Cold Regions Science and Technology 49, 110-133 (2007).

${ }^{18}$ J. Davys, R. Hosking, and A. Sneyd, "Waves due to a steadily moving 
source on a floating ice plate," Journal of Fluid Mechanics 158, 269-287 (1985).

${ }^{19}$ R. M. S. M. Schulkes, R. Hosking, and A. Sneyd, "Waves due to a steadily moving source on a floating ice plate. part 2," Journal of Fluid Mechanics 180, 297-318 (1987).

${ }^{20}$ L. Domino, M. Fermigier, E. Fort, and A. Eddi, "Dispersion-free control of hydroelastic waves down to sub-wavelength scale," EPL (Europhysics Letters) 121, 14001 (2018).

${ }^{21}$ J. D. Joannopoulos, S. G. Johnson, J. N. Winn, and R. D. Meade, Photonic crystals: molding the flow of light (Princeton university press, 2011).

${ }^{22}$ N. Kaina, M. Fink, and G. Lerosey, "Composite media mixing bragg and local resonances for highly attenuating and broad bandgaps," Scientific reports 3, 3240 (2013).

${ }^{23}$ F. Box, D. Vella, R. W. Style, and J. A. Neufeld, "Indentation of a floating elastic sheet: geometry versus applied tension," Proceedings of the Royal Society of London A: Mathematical, Physical and Engineering Sciences 473 (2017), 10.1098/rspa.2017.0335, http://rspa.royalsocietypublishing.org/content/473/2206/20170335.full.pdf.

${ }^{24}$ F. Moisy, M. Rabaud, and K. Salsac, "A synthetic schlieren method for the measurement of the topography of a liquid interface," Experiments in Fluids 46, 1021-1036 (2009).
${ }^{25} \mathrm{~S}$. Wildeman, "Real-time quantitative schlieren imaging by fast fourier demodulation of a checkered backdrop," Experiments in Fluids 59, 97 (2018).

${ }^{26}$ É. Guyon, J.-P. Hulin, and L. Petit, Hydrodynamique physique $3 e$ édition (2012) (EDP sciences, 2012).

${ }^{27}$ Usually, studying a 1D lattice requires a waveguide. Here, because the objects (the perforation) have a size close to the wavelengths it is not possible to confine enough our system in one direction to create a waveguide. Instead, we choose to build a 2D lattice but to only probe it in one direction. Its behavior is then similar to that of a 1D lattice with the same properties.

${ }^{28}$ I. Sersic, M. Frimmer, E. Verhagen, and A. F. Koenderink, "Electric and magnetic dipole coupling in near-infrared split-ring metamaterial arrays," Physical Review Letters 103, 213902 (2009).

${ }^{29}$ E. Shamonina and L. Solymar, "Magneto-inductive waves supported by metamaterial elements: components for a one-dimensional waveguide," Journal of Physics D: Applied Physics 37, 362 (2004).

${ }^{30}$ F. Lemoult, N. Kaina, M. Fink, and G. Lerosey, "Wave propagation control at the deep subwavelength scale in metamaterials," Nature Physics 9, 55-60 (2013).

${ }^{31} \mathrm{~N}$. Kaina, Métamatériaux localement résonants: cristaux photoniques et phononiques sub-longueur d'onde, Ph.D. thesis, Université Paris 7 (2016).

${ }^{32}$ P. de Vries, D. V. van Coevorden, and A. Lagendijk, "Point scatterers for classical waves," Rev. Mod. Phys. 70, 447-466 (1998). 